\title{
PERSPECTIVES ON HIGH-TECH PRODUCT DESIGN FOR BETTER SUPPORTING PRODUCT-SERVICE SYSTEMS
}

\author{
Stelian Brad \\ Research Lab ICPD \\ Technical University of Cluj-Napoca \\ Cluj-Napoca, 400020, Romania
}

\begin{abstract}
Nowadays, product-service systems are seen as enhanced and optimal integrated solutions of products and services with the purpose of improving business sustainability, customer satisfaction, environmental impact and production-consumption balance. The ability to integrate various value added vectors characterizing the concept of product-service system within hightech product design is still a complex task, requiring systematic and comprehensive innovation. This means high-tech product design has to be seen from a broader perspective, as a process emerging from an integrated model of product innovation, marketing innovation, production process innovation and business model innovation over their life-cycles. On this basis, the present paper reveals various key strategies required in new high-tech product design for better supporting product-service systems. A model that establishes priorities for integrating these strategies in the design framework of high-tech products is further proposed.
\end{abstract}

\section{INTRODUCTION}

High-tech industries are key driving forces for economic development (Trott 2004). Companies operating in the high-tech sector are engaged in designing, development and introduction of new products and/or innovative manufacturing processes through the systematic application of scientific and technological knowledge (Mohr, Sengupta, and Slater 2005). The Organization for Economic Cooperation and Development (OECD) identifies high-tech businesses from the perspective of contributions of R\&D expenditures to value added of a particular industry (Kirkpatrick 2002). From this viewpoint, around 40 industries define the high-tech sector (Mohr, Sengupta, and Slater 2005).

Various studies reveal that all high-tech industries share some common characteristics like: market uncertainty, technological uncertainty and competitive volatility (Cagan and Vogel 2002; Mohr, Sengupta, and Slater 2005; Olsen and Sallis 2006; Trott 2004). The key sources of these uncertainties are well-captured by Mohr, Sengupta, and Slater (2005). High levels of uncertainty and volatility generates high business risks. Moreover, the unit-one cost of a high-tech product is very high relative to the costs of reproduction (Coffman and Kaufman 2007; Frutos, Santos, and Borenstein 2004). From these perspectives, designing high-tech products is a challenging process. As a consequence, design competencies have to move towards strategies where the focus shifts from simply designing and selling products to designing and selling an aggregate of products and services that is capable of better fulfilling specific market demands (Baines, Lightfoot, and Evans 2007; Marzini and Vezzoli 2003). This mix of marketable products and services is known in the literature as a product-service system (PSS) (Kang and Wimmer 2008; Tukker and Tischner 2006). PSS is actually a business paradigm aiming to provide sustainability of both consumption and production (Baines, Lightfoot, and Evans 2007; Kang and Wimmer 2008). Some researchers enhanced the definition of PSSs by including an environmental dimension, thus highlighting the fact that PSSs must also lead to lower environmental impacts than traditional business models (Beverungen, Knackstedt, and Muller 2008; Marzini and Vezzoli 2003; Michelini and Razzoli 2004; Partidario, Lambert, and Evans 2007).

PSSs challenge the design process of high-tech products because new and complex dimensions in product definition have to be considered. Thus, products have to be approached from a life-cycle perspective (Komoto, Tomiyama, Nagel, Silvester, and Brezet 2005; Sundin and Lindahl 2008) and incorporating capabilities for fast and cost-effective configuration (Frutos, Santos, and Borenstein 2004). This necessitates rethinking of existent design strategies (Sundin and Lindahl 2008), as well as adoption of new design approaches and methodologies (Maussang, Zwolinski, and Brissaud 2008; Shen and Wang 2008; Thomas, Walter, and Loos 2008), where systematic innovation is required (Williams 2007). 


\section{Brad}

From this broader perspective, integration of various value added vectors characterizing the PSS concept within hightech product design has to be seen as a process emerging from an integrated model of product innovation, marketing innovation, production process innovation and business model innovation over their life-cycles.

Therefore a first purpose of this paper is to introduce the concept of enhanced innovation and to investigate its implications on developing high-tech products. Results are further used to work out a decision-making tool for defining customized design strategies of high-tech products under the PSS paradigm. Key areas of intervention for each particular design case are identified via some priority indexes, by analyzing the design strategies from several viewpoints (i.e. links with the PSS goals, correlations, value weights, innovation gaps).

Conclusions of the theoretical foundation are exemplified on a case study (a software platform for quality cost management). It is shown that commercial success could be increased if several dimensions of innovation are concurrently considered within the design of high-tech products.

\section{ABOUT ENHANCED INNOVATION}

The market success of a high-tech product is significantly associated with the level of innovation which the associated PSS of the respective product incorporates. When superior solutions to critical problems on the market are elaborated, radical PSS innovation occurs (Brad 2008; Mohr, Sengupta, and Slater 2005). Radical innovation must generate either a complete novel product or significant improvements in the performance characteristics of an existent product (Trott 2004). As PSS shows, having a high quality product and attractive prices does not necessarily guaranty product competitiveness; "high value for money" is the new paradigm (Cagan and Vogel 2002).

In most of the cases, when PSS radical innovation is achieved, either a consistent differentiation with respect to competing PSSs is realized or new markets are opened (Brad 2008). Through PSS innovation, high value-added must be created for beneficiary; a PSS innovation that cannot be fully exploited by its target beneficiaries is useless. In addition, a PSS innovation is really radical only when it generates positive effects upon production costs, too, as well as upon the supporting processes over the product life-cycle (Brad 2008). From the perspective of PSS paradigm, besides product innovation, other three dimensions of innovation must be considered when a high-tech product is going to be developed. They refer to production process innovation (also known as infrastructure innovation), marketing innovation and business model innovation (also known as organizational innovation) (Brad 2008; Cagan and Vogel 2002; Coffman and Kaufman 2007).

The consideration of a concurrent approach of all four types of innovation in deriving the design strategies of a product represents a novel way of tackling a PSS. In other words, an integration and early exploitation in product conceptualization and design of the four dimensions of innovation must be considered. Thus, product innovation (PI), marketing innovation (MI), production process innovation (PPI) and business model innovation (BMI), are captured in an aggregated model, which is here called "enhanced innovation" (EI) or "integrated innovation" (II). In this model, dynamics of each type of innovation, as well as the coupled effects of these dynamics should be considered when equating the commercial success of the product. Thus, the life-cycle of the aggregated innovation must be taken into account when developing new products, especially for those in the category of high technology. The proposed generic model of enhanced innovation is illustrated in Figure 1.

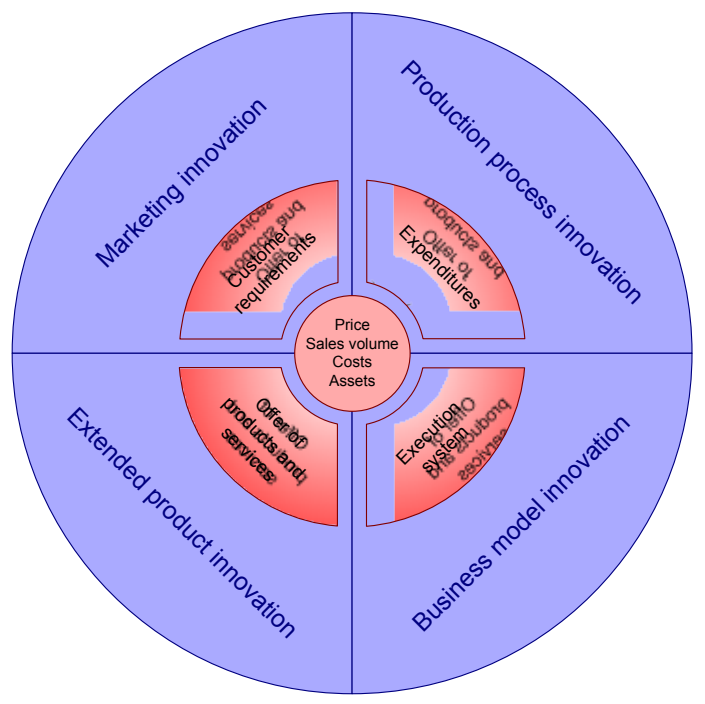

Figure 1: The conceptual model of enhanced innovation 
According to the model of enhanced innovation, when a new product is going to be designed, a clear view about all core blocks of the business system (customers and their requirements, offer in the broad range, production system and expenditures), as well as about their correlations with price, sales volume, costs and assets has to be formulated. But beyond these issues, awareness should be also on how the four axes of innovation will define the PSS's maturity over time, as well as on how to manage the timing between the four axes of innovation such as the business system to evolve in a balanced way (effective and efficient). Without mastering these elements, failures could happen even if the technical innovation incorporated in product is high. In fact, an enhanced innovation should lead to significant differentiation, exciting quality, high value for money, significant reduction of appraisal costs, significant reduction of internal failure costs, significant reduction of lifecycle costs, reduction of manufacturing costs, high attractiveness for venture capital (Brad 2008).

Moreover, it is somehow obvious that product innovation rules in a significant way innovations in marketing, production process and business model. If product is designed with low intrinsic potentials relative to marketing innovation strategies, production process innovation strategies and business model innovation strategies, its market success could be subjected to high risks. In other words, radical innovative ideas in marketing, production and organizational dimensions emerge from product properties, features and characteristics. This aspect should be seen from a life-cycle perspective, too.

In this respect, a new business activity is initiated when a product innovation occurs. As soon as product innovation reaches the viability threshold, product can be launched onto the market. However, without basic innovations in terms of production process and business model, the product alone cannot support the market success. So, during product development phase, innovations in terms of infrastructure, formalization of processes and business capitalization should also occur. More than this, marketing innovation should reach in this period even a higher level of maturity than product innovation to support properly the introduction phase of the product onto the market. When break-even point is reached, innovation must happen consistently in all its four dimensions.

Resources necessary to support the business activity are significantly higher when all aspects of innovation are taken into account. If the business plan lacks of reliable solutions and appropriate effort estimation in terms of marketing innovation, process innovation and business model innovation, financial estimations might look good, whereas the reality would be vice versa. Thus, product innovation and overall business innovation should be permanently kept over the viability threshold curves, otherwise the financial performances and even company's survival could be exposed. Consequently, from a life-cycle perspective, besides a strong articulation of product innovation, clear demonstrations on how actually marketing innovation, production process innovation and business model innovation will evolve in time have to be considered. This will provide more realistic views around the market potential of the new product.

\section{HIGH-TECH PRODUCT DESIGN FROM AN INTEGRATED INNOVATION MODEL PERSPECTIVE}

Taking into account the aspects highlighted in the life-cycle model of enhanced innovation, product design should be viewed from a new angle. Seeing the importance which marketing innovation, production process innovation and business model innovation play in the equation of business competitiveness, the question is how actually these categories of innovations could be supported by product innovation. A reverse approach has to be considered for identifying challenges in high-tech product design for supporting innovations in marketing, technical processes and business processes, thus improving performances of the PSS and providing higher profits for the producing company. In other words, an appropriate concept and appropriate features within the new or improved product could significantly contribute to the definition of highly mature innovations in marketing, infrastructure, organization, etc. Without having the ambition of comprehensively covering all aspects of this problem, the next sub-sections recommend product design strategies under the spectrum of enhanced innovation. Table 1 reveals some characteristics of marketing innovation that visibly influence product design strategies.

Table 1: Implications of Marketing Innovation on High-Tech Product Design Strategies

\begin{tabular}{|l|l|}
\hline Characteristic of marketing innovation & Implications on new high-tech product design (code) \\
\hline Meet market requirements & Customer-oriented design (COD) \\
\hline Deep market segmentation & Design for easy customization (DEC) \\
\hline Proper price policies and pricing strategies & $\begin{array}{l}\text { Design ruled by measurable PSS performance characteristics (MPS) } \\
\text { Consideration of PSS features to support various payment policies (PPP) }\end{array}$ \\
\hline Unique selling points & Novel product features for stringent market needs (SMN) \\
\hline Value orientation & Product capable to define a new market value (NMV) \\
\hline Build a cultural trend around product & Make visible a specific identity for product users (proud to use it) (PUI) \\
\hline Increasing markets and open new markets & $\begin{array}{l}\text { Easy to install and upgrade (EIU) } \\
\text { Easy to learn how to use it (ELU) } \\
\text { Fast prove of value-added and financial benefits (VAF) }\end{array}$ \\
\hline
\end{tabular}


Table 2: Implications of Process Innovation on High-Tech Product Design Strategies

\begin{tabular}{|l|l|}
\hline Characteristic of process innovation & Implications on new high-tech product design (code) \\
\hline Competitive production costs & $\begin{array}{l}\text { Design to cost objective (DCO) } \\
\text { Meet a cost objective for each module and part of the product (COM) }\end{array}$ \\
\hline High process capability & Product designed to facilitate manufacturing quality (FMQ) \\
\hline Minimize poor quality costs & Robust design (ROD) \\
\hline High productivity & Product designed to facilitate manufacturing automation (FMA) \\
\hline Low maintenance and supporting costs & Design for easy maintenance and support (even remotely) (EMS) \\
\hline Adaptation to new product generations & $\begin{array}{l}\text { Reconfigurable design (RED) } \\
\text { Open architecture (OAH) }\end{array}$ \\
\hline High technical flexibility & $\begin{array}{l}\text { Highly modular design (HMD) } \\
\text { Standardized interfaces (SDI) }\end{array}$ \\
\hline
\end{tabular}

Table 3: Implications of Business Innovation on High-Tech Product Design Strategies

\begin{tabular}{|l|l|}
\hline Characteristic of business innovation & Implications on new high-tech product design (code) \\
\hline Business risk minimization & $\begin{array}{l}\text { Configuration design to integrate existent modules onto the market (CDI) } \\
\text { Architectural innovation (AHI) } \\
\text { Modules with well-protected intrinsic functional features (IFF) }\end{array}$ \\
\hline High operating income & $\begin{array}{l}\text { Low operating costs (LOC) } \\
\text { Bring high value-added for customer (price can grow) (HVA) }\end{array}$ \\
\hline High ROI in short time & $\begin{array}{l}\text { Lower development costs (simplicity) (LDC) } \\
\text { Radical technical innovation (uniqueness) (RTI) }\end{array}$ \\
\hline High IRR and NPV & Design for product life-cycle (DLC) \\
\hline Financial sustainability & Design towards directed product evolution (DPE) \\
\hline
\end{tabular}

Tables 2 and Table 3 show some characteristics of process and business innovation that influence product design strategies. The idea emerging from Tables 1, 2 and 3 is that product design cannot be approached only from technical point of view; by contrary, product design has to be driven by market and business environment. The commercial success of the product will be significantly influenced by the capability to "translate" market and business environments into design.

\section{MODELLING DESIGN PRIORITIES FOR HIGH-TECH PRODUCT DESIGN}

Designing a high-tech product by considering all recommendations from Tables 1, 2 and 3 is a complex task and from practical perspective it meets various resource-related constrains (time, human, financial, technological, know-how, localization, etc.). Moreover, at a certain moment, each product reaches a certain position on its S-curve of evolution, which clearly influences the future development strategies (short, medium and long term). In order to guide the decision-making process for high-tech product design from a PSS perspective, with the consideration of design strategies from Tables 1, 2 and 3, a related model is further proposed. To set up the model, the following steps are taken into account:

Define the key objectives of a PSS;

Identify the relationship between PSS objectives and product design strategies;

Determine the level of relationship between PSS objectives and product design strategies;

Determine the value weight of each product design strategy in the framework of PSS;

Determine the relative influence factor of each product design strategy to the other product design strategies;

Determine the innovation priority index with respect to each product design strategy for the analyzed product;

Establish priority actions in product design by combining the resulted information into a decision-making model.

PSS high-level objectives taken into account in this analysis are: to increase the balance between production and consumption (B), to lower the environmental impact (E) and to increase profits (P). The application of AHP method and Saaty scale (Cohen 1995) leads to the following relative ranks of PSS objectives: $R_{B}=7.62, R_{E}=46.19, R_{P}=46.19$. In order to determine these ranks, $\mathrm{E}$ and $\mathrm{P}$ have been considered of the same importance and $\mathrm{B}$ somehow less important than $\mathrm{E}$ and $\mathrm{P}$.

Figure 2 illustrates the relationships between PSS objectives and product design strategies, as well as the value weight of each design strategy in the equation of a commercial successful PSS. A numerical value is associated to each relationship $a_{i k}$, $i=1,2,3 ; k=1, \ldots, 29$, between the PSS objective $i, i=1,2,3$ and the design strategy $k, k=1, \ldots, 29$ (see the design strategies in Tables 1,2 and 3). 


\section{Brad}

\begin{tabular}{|c|c|c|c|c|c|c|c|}
\hline \multirow[b]{3}{*}{ z } & \multirow{3}{*}{ 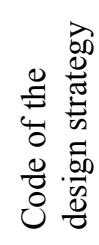 } & \multicolumn{3}{|c|}{ Code of the PSS objective } & \multicolumn{3}{|c|}{ Value weight } \\
\hline & & B & $\mathrm{E}$ & $\mathrm{P}$ & \multirow[t]{2}{*}{ Symbol } & \multirow[t]{2}{*}{ Numeric } & \multirow[t]{2}{*}{$\begin{array}{l}\text { Normalized } \\
\text { to } 100\end{array}$} \\
\hline & & $R_{B}=7.62$ & $R_{E}=46.19$ & $R_{P}=46.19$ & & & \\
\hline 1 & COD & - & $\odot$ & O & $W_{1}$ & 622.86 & 3.5 \\
\hline 2 & DEC & - & $\odot$ & 0 & $W_{2}$ & 622.86 & 3.5 \\
\hline 3 & MPS & $\odot$ & $\triangle$ & - & $W_{3}$ & 484.76 & 2.7 \\
\hline 4 & PPP & 0 & $\odot$ & $\odot$ & $W_{4}$ & 345.72 & 1.9 \\
\hline 5 & SMN & 0 & $\triangle$ & 0 & $W_{5}$ & 530.48 & 3.0 \\
\hline 6 & NMV & 0 & $\triangle$ & 0 & $W_{6}$ & 530.48 & 3.0 \\
\hline 7 & PUI & - & $\triangle$ & $\odot$ & $W_{7}$ & 253.34 & 1.5 \\
\hline 8 & EIU & & 0 & $\odot$ & $W_{8}$ & 554.28 & 3.1 \\
\hline 9 & ELU & & - & $\odot$ & $W_{9}$ & 554.28 & 3.1 \\
\hline 10 & VAF & 0 & $\triangle$ & 0 & $W_{10}$ & 530.48 & 3.0 \\
\hline 11 & $\mathrm{DCO}$ & 0 & - & - & $W_{11}$ & 900.00 & 5.1 \\
\hline 12 & $\mathrm{COM}$ & o & - & $\boldsymbol{0}$ & $W_{12}$ & 900.00 & 5.1 \\
\hline 13 & FMQ & $\odot$ & - & $\boldsymbol{0}$ & $W_{13}$ & 854.28 & 4.8 \\
\hline 14 & ROD & & - & - & $W_{14}$ & 831.42 & 4.6 \\
\hline 15 & FMA & $\odot$ & $\odot$ & o & $W_{15}$ & 577.14 & 3.2 \\
\hline 16 & EMS & - & - & - & $W_{16}$ & 900.00 & 5.1 \\
\hline 17 & RED & - & - & $\odot$ & $W_{17}$ & 622.86 & 3.5 \\
\hline 18 & $\mathrm{OAH}$ & - & - & $\odot$ & $W_{18}$ & 622.86 & 3.5 \\
\hline 19 & HMD & - & $\odot$ & $\odot$ & $W_{19}$ & 300.00 & 1.7 \\
\hline 20 & SDI & 0 & 0 & 0 & $W_{20}$ & 900.00 & 5.1 \\
\hline 21 & CDI & 0 & 0 & $\odot$ & $W_{21}$ & 622.86 & 3.5 \\
\hline 22 & AHI & $\triangle$ & 0 & $\triangle$ & $W_{22}$ & 469.52 & 2.6 \\
\hline 23 & IFF & & & 0 & $W_{23}$ & 415.71 & 2.3 \\
\hline 24 & LOC & & 0 & $\odot$ & $W_{24}$ & 554.28 & 3.1 \\
\hline 25 & HVA & $\odot$ & & 0 & $W_{25}$ & 438.57 & 2.5 \\
\hline 26 & LDC & 0 & $\odot$ & 0 & $W_{26}$ & 622.86 & 3.5 \\
\hline 27 & RTI & & & 0 & $W_{27}$ & 415.71 & 2.3 \\
\hline 28 & DLC & 0 & 0 & 0 & $W_{28}$ & 900.00 & 5.1 \\
\hline 29 & DPE & 0 & 0 & 0 & $W_{29}$ & 900.00 & 5.1 \\
\hline
\end{tabular}

Figure 2: Deployment of PSS objectives into product design strategies

The following values and symbols are used in the matrix from Figure 2 to express relationships between PSS objectives and product design strategies: 0 (blank) - no relationship, $1(\triangle)$ - weak or possible relationship; $3(\odot)$ - medium relationship; 9 (O) - strong relationship (Cohen 1995). Value weights are calculated with formula (1).

$$
W_{k}=R_{B} \cdot a_{k, B}+R_{E} \cdot a_{k, E}+R_{P} \cdot a_{k, P}, \quad \text { where } \quad k=1, . ., n ; n=29 \text {. }
$$

The numerical values of the value weights for the design strategies (considering the set of three PSS objectives and their ranks) are shown in the last columns of Figure 2. The relative influences between the design strategies are determined by means of a correlation matrix, illustrated in Figure 3. The influence coefficients $C_{i j}, i, j=1, \ldots, 29, i \neq j$, take the following values: 0 - no influence; 1 - weak influence; 3 - medium influence; 9 - strong influence (Cohen 1995). In Figure 3, the influence level is symbolized with: blank - no influence; $\bigcirc$ - weak influence; $\mathbf{O}$ - medium influence; $\mathbf{\square}$ - strong influence.

$$
I F_{i}=\sum_{j=1 ; j \neq i}^{n} C_{j i}, \quad i=\overline{1, n} ; n=29
$$




\begin{tabular}{|c|c|c|c|c|c|c|c|c|c|c|c|c|c|c|c|c|c|c|c|c|c|c|c|c|c|c|c|c|c|}
\hline & Oे & $\begin{array}{l}\text { U } \\
\text { प1 }\end{array}$ & $\stackrel{n}{\Sigma}$ & $\vec{a}$ & $\sum_{n}^{Z}$ & $\sum_{Z}^{Z}$ & 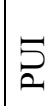 & 穴 & 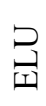 & $\stackrel{5}{5}$ & $\begin{array}{l}0 \\
0 \\
0\end{array}$ & $\sum_{0}$ & $\sum_{I=}^{O}$ & oิ & 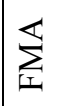 & $\sum_{i=1}^{\infty}$ & 愛 & 焉 & $\sum_{ \pm}$ & $\overrightarrow{\hat{n}}$ & $\overline{0}$ & 焉 & 岌 & ○્ય & 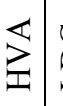 & : & $\vec{E}$ & $\stackrel{\cup}{\vec{\theta}}$ & \\
\hline COD & & $\boldsymbol{\square}$ & 0 & & $\square$ & $\square$ & 口 & $\square$ & $\square$ & 口 & & $\square$ & $\square$ & $\square$ & & $\square$ & 0 & 0 & 0 & & & $\mathbf{0}$ & & $\square$ & $\square$ & & $\square$ & $\boldsymbol{\square}$ & \\
\hline \begin{tabular}{|l} 
DEC \\
\end{tabular} & 0 & & 0 & & & & $\square$ & 0 & & $\square$ & & & & & $\square$ & & $\mathbf{\square}$ & $\mathbf{\square}$ & $\square$ & $\boldsymbol{\square}$ & & $\mathbf{\square}$ & & & 0 & & $\square$ & 0 & \\
\hline \begin{tabular}{|l} 
MPS \\
\end{tabular} & $\mathbf{0}$ & $\boldsymbol{\square}$ & & 0 & & $\square$ & & 0 & & 0 & $\square$ & $\square$ & $\square$ & $\square$ & & & 0 & 0 & & & & 0 & & 0 & 0 & & $\square$ & $\boldsymbol{\square}$ & \\
\hline PPP & $\square$ & 0 & 0 & & 0 & & & $\square$ & 0 & 0 & & & & & & $\mathbf{a}$ & $\mathbf{a}$ & $\mathbf{a}$ & $\square$ & & $\boldsymbol{\square}$ & $\mathbf{\square}$ & $\mathbf{\square}$ & & 0 & & 0 & $\mathbf{\square}$ & \\
\hline \begin{tabular}{|l} 
SMN \\
\end{tabular} & 0 & 0 & 0 & & & $\square$ & 0 & & & 0 & & & & & & & 0 & $\mathbf{a}$ & & & & & $\bar{\square}$ & 0 & $\square$ & & $\square$ & & a \\
\hline NMV & 0 & 0 & 0 & $\mathbf{0}$ & $\square$ & & 0 & & & & & & & & & $\mathbf{0}$ & & & & & & & & & $\bar{\square}$ & & ( & $\mathbf{\square}$ & $\overline{\mathbf{z}}$ \\
\hline PUI & $\mathbf{E}$ & $\mathbf{\square}$ & 0 & & $\boldsymbol{\square}$ & $\mathbf{D}$ & & & & & & & & & & & & & & & & & & & $\mathbf{\square}$ & & $\mathbf{D}$ & & \\
\hline EIU & 0 & & 0 & $\square$ & 0 & & & & 0 & & & & & & & $\square$ & & & $\square$ & 0 & & & & & O & & & $\mathbf{\square}$ & \\
\hline ELU & 0 & & 0 & $\mathbf{0}$ & 0 & & & & & & & & & & & & & & $\mathbf{a}$ & 0 & & & & & O & & & $\mathbf{\square}$ & \\
\hline VAF & $\square$ & $\boldsymbol{\square}$ & & $\mathbf{a}$ & $\square$ & 0 & 0 & & $\square$ & & & & & & & $\mathbf{a}$ & 0 & & 0 & & & & & $\mathbf{\square}$ & $\mathbf{\square}$ & & & $\mathbf{\square}$ & \\
\hline DCO & 0 & & 0 & & & & & & & & & $\square$ & 0 & 0 & $\square$ & & & 0 & $\square$ & $\mathbf{a}$ & $\mathbf{\square}$ & $\mathbf{0}$ & & & $\square$ & $\square$ & $\square$ & & \\
\hline $\mathrm{COM}$ & 0 & & $\mathbf{E}$ & & & & & & & & $\square$ & & 0 & 0 & 0 & & \begin{tabular}{|l|}
0 \\
\end{tabular} & 0 & $\square$ & $\mathbf{\square}$ & & $\mathbf{\square}$ & & & & $\mathbf{\square}$ & $\mathbf{\square}$ & & \\
\hline FMQ & $\square$ & & & 0 & 0 & & & & & & 0 & 0 & & 口 & $\mathbf{D}$ & & $\mathbf{a}$ & & 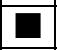 & $\mathbf{a}$ & $\mathbf{\square}$ & & & & & & 0 & $\mathbf{\square}$ & \\
\hline ROD & 0 & & 0 & 0 & & & & & & & $\mathbf{a}$ & $\square$ & $\mathbf{a}$ & & $\boldsymbol{\square}$ & $\mathbf{a}$ & $\mathbf{a}$ & $\mathbf{a}$ & $\mathbf{a}$ & $\mathbf{a}$ & & & & $\square$ & 0 & $\mathbf{\square}$ & 0 & $\mathbf{a}$ & \\
\hline FMA & 0 & & 0 & & & & & & & & $\mathbf{E}$ & $\mathbf{0}$ & & $\mathbf{E}$ & & & $\mathbf{0}$ & & $\mathbf{a}$ & $\mathbf{0}$ & $\mathbf{\square}$ & & & & & & & $\mathbf{\square}$ & \\
\hline EMS & 0 & 0 & & 0 & & & & 0 & 0 & & & & & $\mathbf{\square}$ & & & $\mathbf{a}$ & & $\square$ & $\mathbf{a}$ & $\mathbf{\square}$ & & & 口 & $\mathbf{\square}$ & & $\square$ & $\mathbf{\square}$ & \\
\hline RED & & 0 & 0 & 0 & 0 & & & 0 & & & & & & & & & & $\mathbf{a}$ & $\mathbf{a}$ & $\mathbf{\square}$ & $\mathbf{\square}$ & $\mathbf{\square}$ & & & & & 0 & $\mathbf{a}$ & 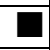 \\
\hline $\mathrm{AH}$ & & $\boldsymbol{\square}$ & & 0 & $\square$ & & & $\square$ & & & $\square$ & 0 & & & & $\mathbf{a}$ & $\mathbf{a}$ & & $\boldsymbol{D}$ & $\mathbf{a}$ & 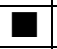 & $\mathbf{\square}$ & & & & $\mathbf{\square}$ & 0 & $\mathbf{a}$ & $\mathbf{\square}$ \\
\hline HMD & & $\boldsymbol{\square}$ & 0 & $\mathbf{a}$ & 0 & & 0 & $\square$ & $\square$ & 0 & 0 & $\square$ & & 0 & $\mathbf{D}$ & $\mathbf{a}$ & $\mathbf{a}$ & $\mathbf{a}$ & & $\mathbf{a}$ & $\mathbf{\square}$ & $\mathbf{\square}$ & 0 & & & $\mathbf{\square}$ & 0 & $\mathbf{\square}$ & $\square$ \\
\hline SDI & & 0 & 0 & $\mathbf{a}$ & & & & $\square$ & & & 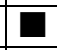 & $\square$ & & 0 & $\mathbf{\square}$ & $\mathbf{a}$ & $\mathbf{a}$ & $\mathbf{a}$ & $\mathbf{a}$ & & $\mathbf{\square}$ & $\mathbf{a}$ & & & & $\mathbf{\square}$ & & $\mathbf{a}$ & $\square$ \\
\hline CDI & & & & $\mathbf{\square}$ & & & & $\mathbf{\square}$ & & & $\mathbf{\square}$ & $\mathbf{\square}$ & & & $\mathbf{\square}$ & $\mathbf{a}$ & $\mathbf{D}$ & $\mathbf{\square}$ & $\mathbf{a}$ & $\mathbf{a}$ & & $\mathbf{\square}$ & $\mathbf{\square}$ & & & $\mathbf{\square}$ & & $\mathbf{\square}$ & $\mathbf{\square}$ \\
\hline AHI & & $\square$ & $\mathbf{\square}$ & $\mathbf{\square}$ & 0 & & 0 & & & & 口 & $\mathbf{D}$ & & & $\mathbf{D}$ & $\mathbf{a}$ & $\mathbf{a}$ & $\mathbf{a}$ & $\mathbf{a}$ & $\mathbf{\square}$ & $\mathbf{\square}$ & & $\bar{\square}$ & & & $\mathbf{\square}$ & 0 & $\mathbf{\square}$ & $\square$ \\
\hline IFF & & & $\boldsymbol{\square}$ & 0 & $\square$ & & 0 & & & & & & & 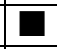 & & $\square$ & $\mathbf{a}$ & & 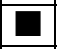 & & $\square$ & & & & & & $\square$ & $\mathbf{\square}$ & \\
\hline LOC & 0 & & & & & & & 0 & & [ & & & & a & & $\square$ & & & & & & & & & & & a & $\mathbf{a}$ & \\
\hline HVA & $\square$ & $\square$ & & $\mathbf{\square}$ & & $\square$ & & 0 & 0 & & & & & 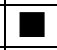 & & $\square$ & $\mathbf{a}$ & $\mathbf{a}$ & 0 & 0 & & & & $\mathbf{\square}$ & & 01 & $\square$ & $\mathbf{\square}$ & $\square$ \\
\hline LDC & & & & & & & & & & & a & $\square$ & & 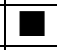 & $\mathbf{\square}$ & & & $\mathbf{a}$ & 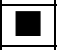 & $\mathbf{\square}$ & 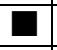 & $\mathbf{a}$ & & & & & $\square$ & & \\
\hline RTI & 0 & & 0 & & $\square$ & $\square$ & 0 & & & & 0 & $\mathbf{\square}$ & & 0 & & $\square$ & $\mathbf{a}$ & $\mathbf{a}$ & 0 & 0 & & & & 0 & & $\mathbf{\square}$ & & $\square$ & L \\
\hline DLC & 0 & & 0 & $\mathbf{a}$ & & & & $\square$ & 0 & $\square$ & & 0 & & 口 & 0 & $\square$ & $\boldsymbol{D}$ & $\mathbf{a}$ & $\boldsymbol{D}$ & $\mathbf{\square}$ & 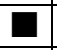 & $\mathbf{a}$ & 0 & $\bar{\square}$ & & & 0 & & $\square$ \\
\hline DPE & & & 0 & & 0 & 0 & 0 & 0 & & & & 0 & & 0 & & $\square$ & $\square$ & $\boldsymbol{D}$ & $\square$ & 0 & & $\square$ & & & & 01 & $\square$ & 口 & \\
\hline Symbol & 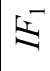 & $\stackrel{2}{N}$ & $\mathbb{I}^{m}$ & 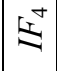 & 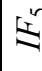 & $\leqslant$ & E & $\leqslant$ & $I^{a}$ & $i^{\circ}$ & \pm & $\mathbb{2}^{2}$ & $\Sigma^{2}$ & \pm & $\underbrace{2}$ & $\stackrel{0}{=}$ & $\leqslant$ & $\underbrace{\infty}$ & $\underbrace{9}$ & $\underbrace{2}$ & $\mathbb{N}^{\bar{N}}$ & $\stackrel{2}{2}$ & $\underbrace{2}$ & $\stackrel{ \pm}{ \pm}$ & $\stackrel{2}{2}$ & 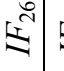 & $\stackrel{\widehat{N}}{\approx}$ & $\mathbb{2}^{\infty}$ & $\underbrace{2}$ \\
\hline Numeric & $\infty$ & $\infty$ & t & $\overline{0}$ & $\hat{\lambda}$ & 8 & iి & $\stackrel{\infty}{\sim}$ & d & $\mathcal{f}$ & ฉ & @ & $\bar{n}$ & ฮิ & $\infty$ & $\stackrel{-}{ \pm}$ & $\bumpeq$ & $\begin{array}{l}\infty \\
\simeq\end{array}$ & $\stackrel{2}{I}$ & $\stackrel{n}{m}$ & $\Xi$ & 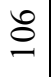 & \& & in & ন & $\infty$ & I & 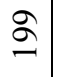 & a \\
\hline $\begin{array}{l}\text { Norm. to } \\
100\end{array}$ & $\stackrel{0}{m}$ & $\vec{m}$ & $\stackrel{n}{i}$ & $\vec{r}$ & $\begin{array}{l}\infty \\
i\end{array}$ & $\stackrel{2}{i}$ & $\stackrel{0}{-}$ & $\hat{i}$ & $\stackrel{+}{-}$ & $\stackrel{n}{-}$ & $\ddot{n}$ & $\stackrel{\circ}{+}$ & $\exists$ & $\ddot{r}$ & $\vec{m}$ & $\stackrel{\nabla}{\dot{n}}$ & $\overrightarrow{i n}$ & $\begin{array}{l}0 \\
i n\end{array}$ & $\begin{array}{l}n \\
0\end{array}$ & $\stackrel{\vartheta}{\dot{\nabla}}$ & $\stackrel{?}{+}$ & $\hat{े}$ & $\stackrel{\nabla}{-}$ & $\vec{i}$ & $\begin{array}{l}\stackrel{0}{i} \\
\stackrel{i}{n}\end{array}$ & $\vec{m}$. & $\vec{n}$ & $\stackrel{?}{r}$ & $\begin{array}{l}\dot{\varphi} \\
\dot{r}\end{array}$ \\
\hline
\end{tabular}

Figure 3: Relative influence factors

Formula (2) shows how the relative influence factors $I F_{i}, i=1, \ldots, 29$, of the design strategies, are calculated. Their numerical values are presented in Figure 3. In order to express the innovation priority with respect to each design strategy for a given product, an index $I G_{i}, i=1, \ldots, 29$, is calculated with formula (3).

$$
I G_{i}=\left(T V_{i}-P V_{i}\right) \cdot \frac{1}{D_{i}}, i=\overline{1, n} ; n=29
$$

In formula (3), $T V_{i}, i=1, \ldots, 29$, is the utility level associated to the target value for the design strategy $i, i=1, \ldots, 29$. The utility level associated to the target value is 10 for each design strategy, on a utility-scale from 0 to 10 . In the same register, $P V_{i}, i=1, \ldots, 29$, is the utility level associated to the current in-house realization of the design strategy $i, i=1, \ldots, 29$, and 


\section{Brad}

$D_{i}, i=1, \ldots, 29$, is the estimated difficulty index to cover the gap $i, i=1, \ldots, 29$, considering current know-how, knowledge and practices, as well as efforts towards new scientific and/or technological discoveries and/or new technical innovations. $P V_{i}, i=1, \ldots, 29$, are quantified on the same utility-scale, from 0 to 10 , whereas $\mathrm{D}_{i}, i=1, \ldots, 29$, are quantified on a five-point Likard difficulty scale (from 1 "no much difficulty" to 5 "extremely high difficulty"). The index of priority $P I_{i}, i=1, \ldots, 29$, associated to each design strategy $i, i=1, \ldots, 29$, for a given product is determined according to formula (4).

$$
P I_{i}=W_{i} \cdot I F_{i} \cdot I G_{i}=\left(R_{B} \cdot a_{i, B}+R_{E} \cdot a_{i, E}+R_{P} \cdot a_{i, P}\right) \cdot\left(\sum_{\substack{k=1 ; \\ k \neq i}}^{n} C_{k i}\right) \cdot\left(T V_{i}-P V_{i}\right) \cdot \frac{1}{D_{i}}, i=\overline{1, n} ; n=29
$$

The 80-20 rule (Brad 2008; Cagan and Vogel 2002; Cohen 1995; Trott 2004) is further applied to select the sub-set of design strategies that should be primarily considered in the future developments of the analyzed product. According to data in Figure 2, $48 \%$ of the design strategies brings $61 \%$ impact on PSS goals. These strategies are highlighted in colored boxes in Figure 2. In this respect, the most effective design strategies look to be: design to cost objective, design for value at module and part levels, design for easy maintenance and support, use of standardized interfaces, design for product life-cycle and design towards directed product evolution. They are closely followed by: design for manufacturability, design for robustness. On the next level of the podium are: customer-oriented design, design for customization, design for reconfiguration, open architecture design, configuration design and design for simplicity.

Figure 3 reveals that $45 \%$ of the design strategies are most influential in the equation of PSS development (over $63 \%$ influence). They are highlighted in colored boxes in Figure 3. In terms of relative influence, the most significant design strategies are: design for life-cycle and design for high modularity. In the second position are included: design for reconfiguration, design for manufacturing automation, radical technical innovation and open architecture design. On the third level are: use standardized interfaces, configuration design, design for value at module and part levels. On the next place are positioned: consideration of architectural innovation, robust design, facilitation of price policies via design features, and consideration of directed product evolution. The model application is further exemplified in a case study.

\section{APPLICATION EXAMPLE}

The prioritization model of high-tech product design strategies towards meeting PSS goals has been tested within an innovation project, coordinated by the author of this paper, dealing with the enhancement of a software tool for quality cost management (a new release). It is not the purpose of this paper to describe in detail the software tool; the focus is on showing how various design strategies presented in Tables 1, 2 and 3 have been selected to define the development framework of the new release of the product. A screenshot showing some windows of the software tool is illustrated in Figure 4.

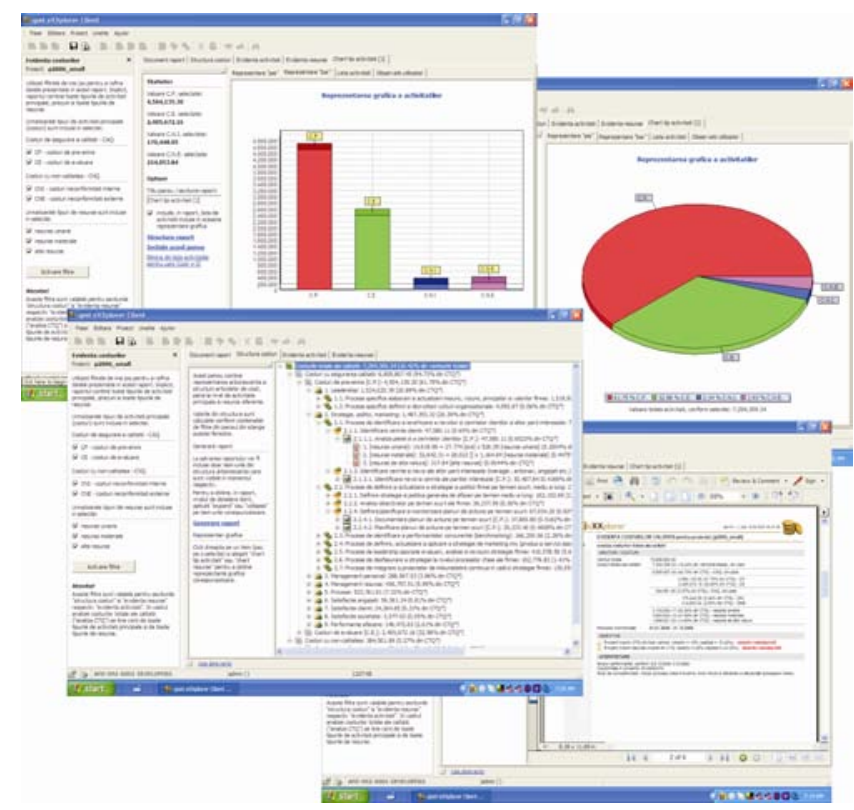

Figure 4: The software product under analysis 
Table 4: Current Characteristics of the Analyzed Software Tool

\begin{tabular}{|l|l|}
\hline Design strategy & Current characteristics \\
\hline COD & Quality planning has been performed, including 4-Phase QFD and detailed SRS \\
\hline DEC & Capability for deep content customization in the set-up phase \\
\hline MPS & After sale services not included in the planning phase \\
\hline PPP & No features included to support various payment policies \\
\hline SMN & An expert system module is included for automatic recommendations based on data collected \\
\hline NMV & Capacity to assess the market value of intangible assets of the business system \\
\hline PUI & Induce a new organizational culture (more responsibility, higher transparency) \\
\hline EIU & Simple installation and upgrading \\
\hline ELU & A training session of 4 days is required \\
\hline VAF & Capacity to compare quality costs with respect to revenues (each trimester) \\
\hline DCO & Low cost platform (much lower than competing products) \\
\hline COM & Software modules were developed using a value engineering methodology and earned-value analysis \\
\hline FMQ & Designed for easy testing \\
\hline ROD & Simple and intuitive model to define quality costs \\
\hline FMA & Easy to be multiplied \\
\hline EMS & Capability for remote monitoring \\
\hline RED & No reconfigurable design \\
\hline OAH & No open architecture \\
\hline HMD & Medium level modular design \\
\hline SDI & No standardized interfaces have been considered \\
\hline CDI & No configuration design has been considered \\
\hline AHI & Classical client-server architecture \\
\hline IFF & No special protection \\
\hline LOC & Capacity to operate the system in network, thus significantly reducing the operational costs \\
\hline HVA & Capacity to cover all sectors in the company; value added dependent by the users' commitment \\
\hline LDC & Small size development team was required (5 people) \\
\hline RTI & Uniqueness given by the expert module for quality cost analysis and planning \\
\hline DLC & No special focus was on designing for product life-cycle \\
\hline DPE & No special focus was on designing for directed product evolution \\
\hline
\end{tabular}

The current characteristics of the product under analysis with respect to the design strategies presented in Tables 1,2 and 3 are highlighted in Table 4 . The calculation of priority index with respect to each design strategy is further revealed in Table 5. According to data in Table 5, the combination of value weight and relative influence factor positions some design strategies in the top of the list. Thus, the most significant design strategy from this perspective is design for product life-cycle. On the second place are situated: design for easy remote maintenance and support, use of standardized interfaces and design for value at the software module level. On the third position are located: reconfigurable design, design for directed product evolution, use of open architecture, robust design, design to meet a cost-objective, and configuration design. On the fourth position are situated: radical technical innovation, modular design, simplicity in design, design for easy customization, customeroriented design, architectural innovation, and facilitation of manufacturing automation.

Considering information from Table 4, utility levels for $P V_{i}, i=1, \ldots, 29$, have been qualitatively appreciated. They are displayed in Table 5. In the same register, the relative difficulties to meet targets have been formulated and displayed in Table 5, too. According to results in Table 5, innovations for further enhancements of the analyzed product should be done with priority on integrating features to support various payment policies considering major PSS performance characteristics, followed by innovations on considering open architecture, use of standardized interfaces and protection of the expert module.

Further innovations should be on configuration design, architectural design, directed product evolution, reconfiguration and life-cycle features. If resources and time allow, enhancements on modularity and remote maintenance and support should be considered.

However, priorities of intervention are guided by the priority indexes $P I_{i}, i=1, \ldots, 29$. According to data in Table 5 , taken into account the current developments of the analyzed product, the model recommends immediate interventions on: inclusion of standard interfaces to link the product with other ERP solutions, design for product life-cycle (with focus on usage phase) and integration of features to support various payment policies considering major PSS performance characteristics. On short-term, priorities should be focused on: open architecture, design for directed product evolution, remote maintenance and 
support, reconfigurable design and configuration design (inclusion of some modules from the market). On medium-term, interventions should be on: architectural innovation and enhanced modularity. On longer-term, developments should be directed towards design for mass customization, increased protection of key product features (e.g. encryption) and automation of development (code reuse).

Table 5: Calculation of Priority Index

\begin{tabular}{|c|c|c|c|c|c|c|c|c|c|c|c|c|c|c|c|c|c|c|c|c|c|c|c|c|c|c|c|c|c|}
\hline & & II & $\stackrel{\tilde{2}}{\xi}$ & $\vec{z}$ & $\sum_{\text {L }}^{Z}$ & $\sum_{Z}$ & 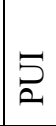 & 袁 & 号 & $\underset{>}{\stackrel{5}{2}}$ & O̊ & 方 & $\stackrel{O}{O}$ & Оิ & $\sum_{i=1}^{\mathbb{I}}$ & $\sum_{I I}^{n}$ & 式 & $\underset{0}{\mathbb{Z}}$ & 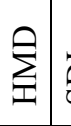 & $\overline{\tilde{\omega}}$ & $\vec{\theta}$ & 焉 & 宦 & $\begin{array}{l}0 \\
0 \\
0 \\
\end{array}$ & 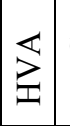 & Uે & $\vec{\sim}$ & $\stackrel{U}{\vec{\Delta}}$ & 跓 \\
\hline$W_{i}$ & 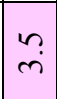 & $n$ & $\hat{i}$ & $\stackrel{q}{-}$ & $\stackrel{0}{\dot{r}}$ & $\stackrel{0}{\dot{r}}$ & $\because$ & $\vec{m}$ & $\vec{m}$ & $\stackrel{\circ}{\dot{r}}$ & $\vec{n}$ & $\vec{n}$ & $\begin{array}{l}\infty \\
\dot{\sim}\end{array}$ & $\begin{array}{l}\dot{\sigma} \\
\dot{\nabla}\end{array}$ & $\stackrel{\sim}{r}$ & $\vec{n}$ & $\ddot{n}$ & $\stackrel{n}{n}$ & $\exists$ & $\vec{n}$ & $\tilde{n}$ & $\begin{array}{l}\stackrel{0}{ } \\
i\end{array}$ & $\stackrel{m}{i}$ & $\vec{r}$ & $\tilde{i}$ & $\ddot{n}$ & $\stackrel{\tilde{i}}{i}$ & $\overrightarrow{n i}$ & $\vec{n}$ \\
\hline$I F_{i}$ & ले & $\vec{m}$ & $\tilde{n}$ & $\hat{n}$ & $\stackrel{\infty}{i}$ & $\stackrel{\sim}{i}$ & $\stackrel{0}{-}$ & $\hat{i}$ & $\stackrel{+}{-}$ & $\stackrel{n}{-}$ & $\stackrel{m}{m}$ & $\stackrel{\circ}{+}$ & $\exists$ & $\hat{r}$ & $\vec{m}$ & $\vec{n}$ & in & $\begin{array}{l}0 \\
\dot{n}\end{array}$ & $\tilde{0}$ & $\stackrel{\vartheta}{+}$ & $\stackrel{m}{+}$ & $\stackrel{े}{\ddot{r}}$ & $\stackrel{\oplus}{-}$ & $\vec{i}$ & $\begin{array}{l}0 \\
i\end{array} \mid$ & $\vec{m}$ & $\vec{n}$ & $\stackrel{?}{r}$ & $\stackrel{0}{\dot{m}}$ \\
\hline$W_{i} \cdot I F_{i}$ & n & $\begin{array}{l}\infty \\
\infty \\
0 \\
0\end{array}$ & $\overrightarrow{\widehat{\sigma}}$ & $\hat{O}$ & $\begin{array}{l}0 \\
+ \\
\infty\end{array}$ & $\begin{array}{l}8 \\
6 \\
6\end{array}$ & $\stackrel{n}{n}$ & $\begin{array}{l}2 \\
\infty \\
\infty\end{array}$ & 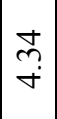 & $\begin{array}{l}\stackrel{0}{n} \\
\dot{r}\end{array}$ & $\mid \begin{array}{l}0 \\
\infty \\
0 \\
-\end{array}$ & $\begin{array}{l}0 \\
\dot{+} \\
\dot{0}\end{array}$ & $\begin{array}{l}\infty \\
i \\
i \\
i\end{array}$ & $\begin{array}{l}\text { ํ. } \\
\text { I }\end{array}$ & $\begin{array}{c}\alpha \\
\alpha\end{array}$ & 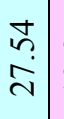 & 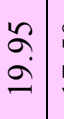 & 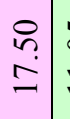 & $\begin{array}{l}n \\
\stackrel{n}{=} \\
=\end{array}$ & $\begin{array}{l}\stackrel{\partial}{\sim} \\
\dot{\sim}\end{array}$ & $\begin{array}{l}n \\
0 \\
i\end{array}$ & $\begin{array}{l} \pm \\
\stackrel{0}{0}\end{array}$ & $\begin{array}{l}\tilde{N} \\
\dot{\sim}\end{array}$ & $\bar{n}$ & 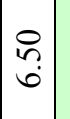 & $\begin{array}{c}n \\
\infty \\
0 \\
0\end{array}$ & $\begin{array}{l}\cong \\
\Xi \\
=\end{array}$ & $\underset{\sim}{\hat{n}}$ & $\begin{array}{l}0 \\
m \\
\infty \\
\infty\end{array}$ \\
\hline$T V$ & 9 & $\begin{array}{l}\stackrel{0}{0} \\
\stackrel{0}{0}\end{array}$ & $\stackrel{0}{0}$ & $\stackrel{0}{0}$ & $\stackrel{0}{0}$ & $\stackrel{0}{0}$ & $\stackrel{0}{0}$ & $\stackrel{0}{0}$ & $\stackrel{0}{0}$ & $\stackrel{0}{0}$ & $\stackrel{0}{0}$ & $\stackrel{\circ}{0}$ & $\stackrel{0}{\circ}$ & $\stackrel{\circ}{\circ}$ & $\stackrel{\circ}{\circ}$ & $\stackrel{\circ}{\circ}$ & $\stackrel{\circ}{\circ}$ & $\stackrel{\circ}{\circ}$ & $\stackrel{\circ}{\circ}$ & $\stackrel{\circ}{\circ}$ & $\stackrel{\circ}{\circ}$ & $\stackrel{\circ}{\circ}$ & $\stackrel{\circ}{\circ}$ & $\stackrel{0}{0}$ & $\stackrel{0}{0}$ & $\stackrel{0}{0}$ & $\stackrel{\circ}{\circ}$ & $\stackrel{\circ}{\circ}$ & \\
\hline$P V_{i}$ & $\begin{array}{l}0 \\
\infty \\
\infty\end{array}$ & $\stackrel{0}{\circ}$ & $\because$ & $\because$ & $\ddot{a}$ & $\ddot{0}$ & $\begin{array}{l}0 \\
\infty\end{array}$ & $\ddot{0}$ & $\dot{a}$ & $\stackrel{0}{a}$ & $\ddot{a}$ & $\begin{array}{l}0 \\
a\end{array}$ & 0 & $\stackrel{0}{\infty}$ & $\stackrel{0}{a}$ & $\stackrel{\circ}{\circ}$ & $\stackrel{0}{0}$ & $\ddot{0}$ & $\stackrel{0}{i}$ & $\because$ & $\ddot{\circ}$ & ir & $\ddot{0}$ & $\stackrel{0}{i}$ & $\infty$ & $n$ & $\tilde{a}$ & $\ddot{0}$ & $\ddot{0}$ \\
\hline$D_{i}$ & 2 & 3 & 3 & 1 & 4 & 4 & 5 & 3 & 2 & 2 & 4 & 4 & 4 & 5 & 1 & 2 & 5 & 3 & 3 & 3 & 4 & 2 & 3 & 4 & 4 & 5 & 5 & 5 & 4 \\
\hline$I G_{i}$ & 18 & $\underset{-}{8}$ & $m$ & $\begin{array}{l}8 \\
\dot{0} \\
-\end{array}$ & $\stackrel{2}{2}$ & {$\left[\begin{array}{l}n \\
\vdots \\
0\end{array}\right.$} & 守. & ?n & $\begin{array}{l}0 \\
n \\
0\end{array}$ & $\begin{array}{l}0 \\
n \\
0 \\
0\end{array}$ & 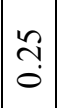 & 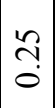 & $\stackrel{8}{\circ}$ & 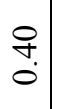 & $\stackrel{8}{-}$ & $\stackrel{\circ}{\stackrel{n}{-}}$ & $\begin{array}{l}\stackrel{8}{0} \\
i\end{array}$ & $\stackrel{m}{\oplus}$ & $\stackrel{\bullet}{\bullet}$ & $\stackrel{m}{m}$ & $\begin{array}{l}0 \\
n \\
i\end{array}$ & $\begin{array}{l}\stackrel{n}{i} \\
i\end{array}$ & $\stackrel{m}{m}$ & $\stackrel{n}{2}$ & in & $\frac{0}{0}$ & $\frac{0}{0}$ & $\begin{array}{l}8 \\
\text { i }\end{array}$ & in \\
\hline$P I_{i}$ & $\begin{array}{l}\stackrel{0}{n} \\
?\end{array}$ & 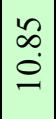 & 我 & 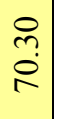 & $\stackrel{0}{i}$ & {$\left[\begin{array}{r}n \\
\hdashline \\
-\end{array}\right.$} & $\stackrel{8}{0}$ & $\hat{a}$ & $\frac{\vec{i}}{i}$ & $\begin{array}{c}\sim \\
i \\
i\end{array}$ & $\vec{\sim}$ & $\mid \begin{array}{l}0 \\
\dot{n}\end{array}$ & $\begin{array}{l}\infty \\
i \\
i \\
i\end{array}$ & $\begin{array}{l}0 \\
\infty \\
0 \\
0\end{array}$ & $\tilde{\alpha}$ & $\overrightarrow{\tilde{\sigma}}$ & ڤે & $\begin{array}{l}\infty \\
\sim \\
\infty \\
i n\end{array}$ & $\begin{array}{l}\dot{m} \\
\infty \\
\infty\end{array}$ & $\begin{array}{c}\tilde{N} \\
\infty \\
\infty\end{array}$ & $\begin{array}{l}\hat{n} \\
\hat{n}\end{array}$ & $\begin{array}{l}n \\
\tilde{n} \\
\end{array}$ & 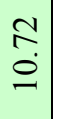 & $\left|\begin{array}{l}\infty \\
\infty \\
\dot{\tau}\end{array}\right|$ & $\stackrel{2}{n}$ & $\stackrel{\infty}{\circ}$ & $\Xi$ & $\begin{array}{l}0 \\
+ \\
\dot{T}\end{array}$ & $\begin{array}{l}8 \\
\text { in } \\
\text { fon }\end{array}$ \\
\hline
\end{tabular}

A qualitative analysis of the current software product against the results revealed in Table 5 was done by a focus group of stakeholders (current customers, developers, product managers, and quality experts). The conclusion was the model led to reliable results, this being a first indicator of its validity. In terms of validity, a few remarks are further necessary.

A first important remark is about the use of the normalized values for $W_{i}$ and $I F_{i}$ in Table 5 . The reason is to ensure a balance between the scales used for these factors and the scale used for $I G_{i}$. Thus, all factors which equate the prioritization model have a balanced significance in the decision-making process.

The second remark is related to the numerical values selected to express the level of relationship (see Figure 2) and the level of influence (see Figure 3). The combination 0-1-3-9, beyond the fact it is widely used in practice, brings more robustness in analysis than other combinations (e.g. 0-1-2-3, 0-1-3-5). This is because all strong relationships and influences are much more evident than medium or weak relationships and influences, thus some errors in expert judgment will not significantly affect the final conclusions.

The third remark is about the scale selected for $T V_{i}$ and $P V_{i}$. The reason to consider the scale from 0 to 10 was justified from the perspective of intuitiveness in judging the level of achievement with respect to the target value. Design strategies are complex and aggregated descriptors of performance; they are not simple metrics, therefore appreciations are strongly qualitative in nature. In most of the cases when a jury has to decide about the performance of a complex, fuzzy action (see the analogy with some individual sports like gymnastic, dance, etc.) a scale from 0 to 10 is used, because it is somehow more accessible and intuitive with respect to human judgment.

The fourth remark is about the scale selected for expressing $D_{i}$. Countless opinions consider that when people think in terms of difficulty levels (but not only), a five-point scale is the most robust one (it induces less errors). Therefore, market surveys usually operate with a five-point Likard scale.

Moreover, the scale used for $T V_{i}-P V_{i}$ is of the same magnitude as the scale used for $D_{i}$, thus the result $\left(I G_{i}\right)$ comes up at a similar magnitude with the normalized values of $W_{i}$ and $I F_{i}$. 


\section{CONCLUSIONS AND FURTHER RESEARCHES}

The purpose of this paper has been to analyze high-tech product design from the perspective of product-service systems in the framework of integrated innovation paradigm and to define a model for prioritizing design strategies for increasing the market impact of product. Seen product innovation in strong connection with marketing innovation, production process innovation and business model innovation, a set of twenty nine design strategies is highlighted in this paper for supporting product development with respect to the major goals of product-service systems.

Because the design process of high-tech products is a complex task, and because the design strategies are complex, too, selection of the most impacting design strategies for short, medium and long term horizon, for each particular case is very important from practical perspective. A decision-making model for this purpose is elaborated in the paper. It considers value weights, influence factors and innovation indexes to calculate the priority factor for each design strategy, tailored to the context of each particular product. The model visualizes the implications of each design strategy in meeting the generic goals of product-service systems and provides means to customize the design path for each specific case under consideration.

The research in this paper is also subjected to some limitations which open new opportunities for further works. In this respect, the consideration of a restricted set of PSS goals as initial inputs in the model would be seen by some readers as a limitation. However, the methodology here proposed allows a fast and easy enhancement of the model with new inputs. Moreover, the selected numerical values for expressing the relationships and influences would be criticized by some readers. It was explained the reason for their selection, but it is admitted that researches could be further directed in this zone in order to refine the analysis (e.g. using fuzzy logic). The set of design strategies could be also subjected to enhancements, too.

Beyond of all these aspects, the model proposed in the paper provides a fast and practical tool to establish an appropriate design pattern of high-tech products from an extended perspective, of product-service systems.

\section{ACKNOWLEDGMENTS}

Financial support from the European Commission, the FP7 research grant TECH IT EASY, no. 232410, is acknowledged with gratitude.

\section{REFERENCES}

Baines, T. S., H. W. Lightfoot, and S. Evans. 2007. State-of-the-art in product-service systems. Journal of engineering manufacture 221(10): 1543-1552.

Beverungen, D., R. Knackstedt, and O. Muller. 2008. Developing service oriented architectures for product-service systems a conceptual approach and its application for the recycling of electronic equipment. Wirtschaftsinformatik 50(3): 220234.

Brad, S. 2008. Complex system design technique: a systematic approach of innovation in a complex world. Cluj-Napoca: Dacia, Inc.

Cagan, J., and C. Vogel. 2002. Creating breakthrough products, Upper Saddle River, New Jersey: Pearson Prentice Hall, Inc.

Coffman, B., and M. Kaufman 2007. Innovation and transformation: a lifecycle model. Available via $<$ http: / / www. innovationlabs.com> [accessed April 4, 2007].

Cohen, L. 1995. Quality function deployment: how to make QFD work for you. Berkeley, California: Addison Wesley Longman, Inc.

Frutos, J. D., E. R. Santos, and D. Borenstein. 2004. Decision support system for product configuration in mass customization environments. Concurrent engineering - research and applications 12(2): 131-144.

Kang, M. J., and R. Wimmer. 2008. Product service systems as advanced system solutions for sustainability. In Proceedings of the EU-Korea Conference on Science and Technology, ed. S. D. Yoo, 191-199. Heidelberg: Springer-Verlag Berlin.

Kirkpatrick, D. 2002. The online grocer version 2.0. Fortune 25: 217-222.

Komoto, H., T. Tomiyama, M. Nagel, S. Silvester, and H. Brezet. 2005. Life cycle simulation for analyzing product service systems. In Proceedings of the 4th International Symposium of Environmentally Conscious Design and Inverse Manufacturing, ed. R. Yamamoto, Y. Furukawa, H. Hoshibu, P. Eagan, H. Griese, Y. Umeda, and K. Aoyama, 386-393. Tokyo: Japan Society of Precision Engineering.

Maussang, N., P. Zwolinski, and D. Brissaud. 2008. Evaluation of product-service systems during early design phase. In $41^{s t}$ CIRP Conference on Manufacturing Systems, ed. M. Mitsuishi, K. Ueda, and F. Kimura, 547-552. Tokyo: University Tokio.

Marzini, E., and C. Vezzoli. 2003. A strategic design approach to develop sustainable product service systems: examples taken from the "environmentally friendly innovation" Italian prize. Journal of cleaner production 11(8): 851-857. 
Michelini, R. C., and R. P. Razzoli. 2004. Product-service for environmental safeguard: a metrics to sustainability. Resources conservation and recycling 42(1): 83-98.

Mohr, J., S. Sengupta, and S. Slater. 2005. Marketing of high-technology products and innovations, Upper Saddle River, New Jersey: Pearson Prentice Hall, Inc.

Olsen, N. V., and J. Sallis. 2006. Market scanning for new service development. European journal of marketing 40(5-6): 466-484.

Partidario, P. J., J. Lambert, and S. Evans. 2007. Building more sustainable solutions in production-consumption systems: the case of food for people with reduced access. Journal of cleaner production 15(6): 513-524.

Shen, J., and L. Y. Wang. 2008. A methodology based on fuzzy extended quality function deployment for determining optimal engineering characteristics in product-service system design. In Proceedings of 2008 IEEE International Conference on Service Operations and Logistics, and Informatics, ed. Beijing Jiaoton Univ., 331-336. Beijing: IEEE Intelligent Transport Systems Society.

Sundin, E., and M. Lindahl. 2008. Rethinking product design for remanufacturing to facilitate integrated product service offerings. In 16th IEEE International Symposium on Electronics and the Environment, ed. IEEE, 263-268. San Francisco: IEEE Computer Society.

Thomas, O., P. Walter, and P. Loos. 2008. Product-service systems: design and usage of the engineering methodology. Wirtschaftsinformatik 50(3): 208-219.

Trott, P. 2004. Innovation management and new product development, Upper Saddle River, New Jersey: Pearson Prentice Hall, Inc.

Tukker, A., and U. Tischner. 2006. Product-services as a research field: past, present and future. Reflections from a decade of research. Journal of cleaner production 14(17): 1552-1556.

Williams, A. 2007. Product service systems in the automobile industry: contribution to system innovation? Journal of cleaner production 15(11-12): 1093-1103.

\section{AUTHOR BIOGRAPHY}

STELIAN BRAD is Full Professor at the Technical University of Cluj-Napoca, Romania, leading the research group on Competitive Engineering in Design and Development. He is also the Director of the Department of Research, Development and Innovation Management of the same university. He got a BSc in Engineering Design (Honors degree) at the Technical University of Cluj-Napoca, a MSc in Energy Management (Honors degree) at the International Technological University Paris, a $\mathrm{PhD}$ in Robotics (Magna Cum Laude) at the Technical University of Cluj-Napoca, with co-tutelage from University Stuttgart. Currently, he is running the second $\mathrm{PhD}$ in Economics at the Academy of Economic Sciences, Bucharest, with focus on process improvement and innovation in SMEs. His research interests include competitive engineering, process innovation, product innovation, intelligent industrial robotics. His email address is <stelian.brad@staff.utcluj.ro>. 